\title{
Bioinformatic Analysis of the Effect of the Sirtuin Family on Differentiated Thyroid Carcinoma
}

\author{
Lijun Yao $\mathbb{D}$ and Yinhua Wang $(\mathbb{D}$ \\ Department of Oncology, Suzhou Ninth People's Hospital, Suzhou 215200, China \\ Correspondence should be addressed to Yinhua Wang; denise914@sina.com
}

Received 25 May 2021; Accepted 10 December 2021; Published 30 January 2022

Academic Editor: Sheba Mohankumar

Copyright (C) 2022 Lijun Yao and Yinhua Wang. This is an open access article distributed under the Creative Commons Attribution License, which permits unrestricted use, distribution, and reproduction in any medium, provided the original work is properly cited.

\begin{abstract}
A growing body of experimental evidence suggests that sirtuins (SIRTs) are associated with tumorigenesis in differentiated thyroid cancer (DTC). Nevertheless, the involvement of SIRTs in the pathogenesis of DTC and their clinical value remain ill-defined and should be thoroughly examined. We explored the transcription of SIRTs and survival data of patients with DTC by the systematic utilization of bioinformatics to analyze data of publicly accessible databases including Oncomine, cBioPortal, Kaplan-Meier Plotter, Gene Expression Profiling Interactive Analysis (GEPIA), Protein Atlas, LinkedOmics, and GSCALite. The examination of gene expression profiles showed that SIRT2, SIRT3, SIRT4, SIRT5, and SIRT6 were downregulated in DTC tissues compared with the normal thyroid tissues. The decreased expression levels of SIRT2, SIRT4, and SIRT5 were correlated with advanced tumor stages. The survival results showed that the increased SIRT4 mRNA expression level was associated with improved overall survival (OS) in the DTC patients. In addition, patients with DTC with high SIRT2, SIRT3, SIRT4, and SIRT5 mRNA levels had higher disease-free survival (DFS). These results showed that SIRT2, SIRT3, SIRT4, SIRT5, and SIRT6 are potential targets for precise treatment of DTC patients and that SIRT2, SIRT3, SIRT4, and SIRT5 are novel potential biomarkers for the prognosis of DTC.
\end{abstract}

\section{Introduction}

Thyroid cancer is classified among the most widespread malignant tumors that occur in the endocrine system. The global incidence of thyroid cancer, especially differentiated thyroid cancer (DTC), has been steadily amplified in recent years. DTC is the major type of thyroid cancer and encompasses papillary thyroid cancer (PTC) and follicular thyroid cancer (FTC) [1]. PTC accounts for about $80 \%$ of thyroid cancer, while FTC accounts for about $15 \%$ within the total of $95 \%$ DTC patients [2]. Currently, the main treatments for DTC include surgery, postoperative assisted ablation, thyroid stimulating hormone (TSH) inhibition therapy, and targeted molecular therapy [3]. Targeted molecular therapy has become a new approach for the treatment of advanced thyroid cancer $[4,5]$. Studies have shown that $R E T$, RAS, BRAF, and VEGF are closely related to the pathogenesis of thyroid cancer, which lay a foundation for targeted molecular therapy [6-8]. About $70 \%$ of PTC is caused by BRAF mutations, RAS mutations and RET/PTC gene rearrangements [9]. In FTC, RAS point mutations, and $P P A R \gamma / P A X 8$ gene rearrangements produce the PPFP fusion gene, the most common oncogene alteration, and PTEN deletion/mutation; PIK3CA mutation and IDH1 mutation are also responsible for FTC [10-13]. Small molecule inhibitors targeting these signaling kinases have become a hot spot for targeted therapies. Due to the heterogeneity of tumors, the use of biomarkers for predicting targeted therapies has some limitations. Therefore, new biomarkers are needed in this field to effectively enhance prognosis and individualized treatment.

Sirtuins (SIRTs) are deacetylases that are highly conserved from bacteria to humans. To date, there are seven recognized members of the human SIRT family which are numbered in order of their discovery into SIRT1-7. X-ray crystal diffraction revealed that multiple members of the SIRT family contain one small domain made of approximately 270 amino acids and a large domain [14]. The large 
domain is mainly constituted of the Rossmann folds while the small domain encompasses a zinc finger structure [15]. The SIRT family has a deacetylase activity and ADPnuclease transferase activity, and the deacetylation mediated by SIRTs is characterized by the transfer of the acetyl group to the ADP-ribosyl of NAD [16]. SIRTs mediate both catalytic activities of deacetylation and NAD cleavage. The ADP-ribosyltransferase activity of SIRTs is the transfer of ADP-ribose from NAD to acetylated proteins [16]. SIRTs are of great importance in clinical medicine and basic research. SIRTs are significantly dysregulated in many malignancies challenging human health, in particular colorectal cancer, prostate cancer, ovarian cancer, lung cancer, breast cancer, and thyroid cancer [16-19]. The interactions between mammalian SIRTs and FOXO/PGC-1 $\alpha, \mathrm{Ku} 70, \mathrm{NF}-$ $\kappa B, p 53$, and other proteins modulate cellular metabolism, cellular stress response, aging, and apoptosis [20, 21]. SIRTs are thought to have complex and unique features in human DTC $[10,11]$. SIRT1 was shown to participate in the regulation of $p 21$ and Bax-related molecular events via the SIRT1Foxp3 pathway in PTC cells [22]. Other research groups have found that by inhibiting ERK and $\mathrm{Mcl}-1$, SIRT6 silencing can downregulate the invasiveness of PTC cells in vitro. Compared with normal thyroid cancer cells, the expression of SIRT7 was significantly increased in DTC, and the overexpression of SIRT7 and SIRT1 conferred resistance to DTC cells [23, 24]. In addition, some researchers have found that the SIRT family plays a pivotal role by downregulating the expression pattern of the tumor-suppressor gene $A R H I$ in thyroid cancer cells [25]. But so far, which SIRT family is activated or inhibited and the unique function of SIRTs in thyroid cancer remain to be absolutely deciphered [16]. Dysregulation of the SIRTs and its relationship with clinical and pathological traits and the predictive value have been conveyed in human thyroid cancer. With the advent of microarray and nextgeneration sequencing technology, revolutionary advances have become an important part of biological and biomedical research $[26,27]$ and have allowed data mining using bioinformatical approaches. Nevertheless, to the best of our knowledge, bioinformatical approaches have not been used to figure out the link between the SIRTs and DTC [28].

Herein, based on publicly available databases, we analyzed in detail different SIRTs in patients with DTC to examine their expression changes, probable function, and prognostic value of SIRT family in DTC.

\section{Materials and Methods}

2.1. Patients. This study was performed based on bioinformatics analysis of The Cancer Genome Atlas (TCGA) data stored in different databases, namely Oncomine, cBioPortal, Kaplan-Meier Plotter, The Gene Expression Profiling Interactive Analysis (GEPIA), Protein Atlas, LinkedOmics, and GSCALite. No particular approval was needed, and the study followed TCGA policies.

2.2. Oncomine. Oncomine is a database containing microarray expression data for cancers and integrated data-mining platform (http://www.oncomine.org/). Oncomine was employed for analyzing and visualizing the expression levels of genes in the SIRT family members in diverse cancers following the online instructions. The mRNA levels of SIRTs in normal and cancer tissues were compared, and Student's $t$ test was used for assessing the difference between both groups. The significant differences were declared at $p<0.05$.

2.3. GEPIA. GEPIA is an interactive online platform for mining the RNA sequencing data from the GenotypeTissue Expression (GTEx) and TCGA projects (http://gepia .cancer-pku.cn/). GEPIA was used for analyzing the expression profiles of SIRTs in DTC and its pathological stages following the default settings online. GEPIA was also used for survival analysis based on the SIRTs.

2.4. The Kaplan-Meier Plotter. The Kaplan-Meier Plotter (http://www.kmplot.com/) was used to analyze the OS and DFS of patients with DTC. The samples were grouped into high expression and low expression groups relatively to the median expression. The Kaplan-Meier Plotter was used for generating the survival plot containing the $\log$ rank $p$ value and the hazard ratio (HR) with $95 \%$ confidence intervals (CIs).

2.5. cBioPortal. TCGA database contains genomic and clinical data on more than 30 cancer types. Samples from TCGATHCA dataset were chosen and used for the analysis of SIRTs using cBioPortal (http://www.cbioportal.org). Genetic variations were analyzed by selecting copy number alterations (CNAs) and mutations as selected molecular profiles.

2.6. Human Protein Atlas. Immunostaining images of SIRTs in human DTC tissues compared to nontumorous thyroid tissues were obtained from the Protein Atlas database (https://www.proteinatlas.org).

2.7. LinkedOmics. The LinkedOmics database is a multiomics tool for the interpretation of attribute associations between the existing cancer databases [29]. It was used for association analysis between the SIRTs and other genes in the DTC RNA-Seq data. The analysis was performed online following the instructions displayed on the platform (http:// www.linkedomics.org).

2.8. Functional Enrichment Analysis. GO and KEGG functional enrichment analyses were performed to uncover the functions prominently associated with the SIRTs and their coregulated genes using the ClusterProfiler package in the R software.

\section{Results}

3.1. Genomic Profiles of SIRTs. The genomic profiles of SIRT family members in patients with DTC were assessed utilizing the Oncomine, GEPA, and Human Protein Atlas databases. The transcriptional expression of SIRTs between various normal and cancer tissues was explored by data analysis in the Oncomine database (Figure 1). The results indicated that SIRTs were diversely expressed in different cancer types. Here, DTC was classified to head and neck cancer. Six unique analyses showed significant differences for SIRT1 in 


\begin{tabular}{|c|}
\hline $\begin{array}{c}\text { Analysis type } \\
\text { by cancer }\end{array}$ \\
\hline Bladder cancer \\
\hline Brain and CNS cancer \\
\hline Breast cancer \\
\hline Cervical cancer \\
\hline Col rectal cancer \\
\hline Esophageal cancer \\
\hline Gastric cancer \\
\hline Head and neck cancer \\
\hline Kidney cancer \\
\hline Leukemia \\
\hline Liver cancer \\
\hline Lung cancer \\
\hline Lymphoma \\
\hline Melanoma \\
\hline Myeloma \\
\hline Other cancer \\
\hline Ovarian cancer \\
\hline Pancreatic cancer \\
\hline Prostate Cancer \\
\hline Sarcoma \\
\hline Significant unique analyses \\
\hline Total unique analyses \\
\hline
\end{tabular}

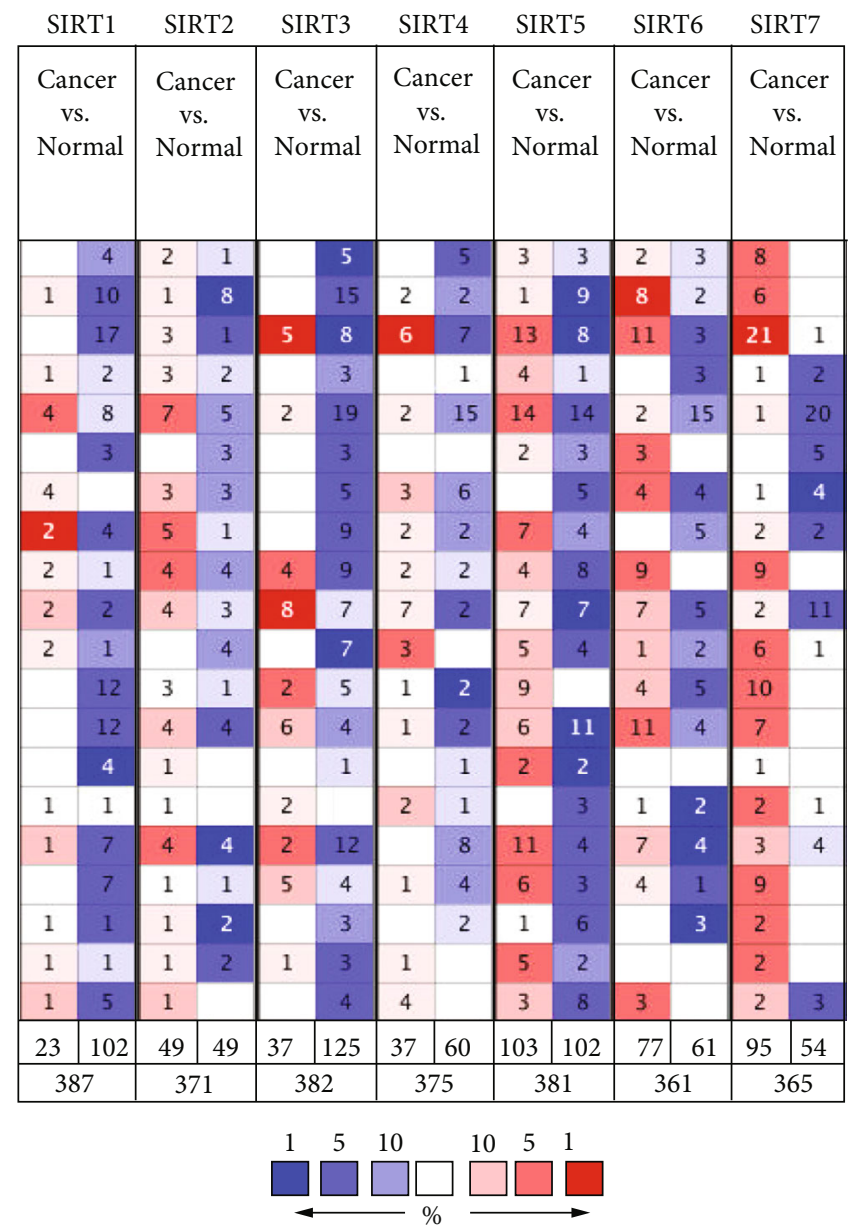

FIGURE 1: The transcription levels of SIRTs in different types of cancers (Oncomine). Red color represents elevated expression, and blue color represents reduced expression. The depth of the color represents the best gene rank percentile.

head and neck cancer, of which two were upregulated and four were downregulated. Similar results were observed for SIRT3 and SIRT4. The same expression trends were also observed in the other cancer types. The expression levels of SIRT2 in head and neck cancer were upregulated in five unique analyses and downregulated in one unique analysis. Similar results were observed for SIRT5, including 7 upregulations and 4 downregulations. Compared with normal tissues, SIRT6 mRNA expression levels in head and neck cancer were significantly decreased in five unique analyses. The expression levels of SIRT7 were elevated in the majority of analyses across all types of cancer. For head and neck cancer, however, SIRT7 was upregulated in two unique analyses and downregulated in two unique analyses.

Next, the GEPIA database was exploited for examining the expression of the SIRTs in DTC samples in comparison with the normal tissues at mRNA level. The scatter diagram and the box plot of the expression levels of SIRTs are reported in Figures 2(a) and 2(b), respectively. 512 DTC samples and 337 normal tissues were selected. The results displayed that the expression levels of SIRT2, SIRT3, SIRT4, SIRT6, and SIRT7 in DTC tissues were lower than those in normal tissues (Figures 2(a) and 2(b)). Immunohistochemis- try (IHC) analysis from the Protein Atlas database was done to assess the protein expression of SIRT proteins in DTC tissues. There was moderate or weak immunoreactivity of SIRTs $1,3,4,5,6$, and 7 in DTC tissues while their corresponding immunoreactivity was relatively weaker in normal tissues (Figure 2(c)). SIRT2 was not detected in both DTC and normal tissues. We also analyzed the expression of the SIRT family in different tumor stages in DTC. The decreased expression of SIRT2, SIRT4, and SIRT5 was significantly associated with advanced stages of DTC, while the expression levels of SIRT1, SIRT3, SIRT6, and SIRT7 groups did not differ significantly between the DTC stages (Figure 3(a)). Pearson's correlation was also performed to evaluate whether there was a relationship between SIRTs in DTC. The results showed that there were significantly positive correlations observed between SIRT1 and SIRT2/3/5/7, SIRT2 and SIRT3/4/5/6/7, SIRT3 and SIRT4/5/6/7, SIRT4 and SIRT5/7, SIRT5 and SIRT7, and SIRT6 and SIRT7 (Figure 3(b), shown in blue). SIRT1 had a negative correlation with SIRT6 $(p=0.028)$. No significant correlations were found between SIRT1 and SIRT4, SIRT4 and SIRT6, or SIRT5 and SIRT6. 32 (2\%) of selected patients (1503) had altered genes, including missense mutation (SIRT4 


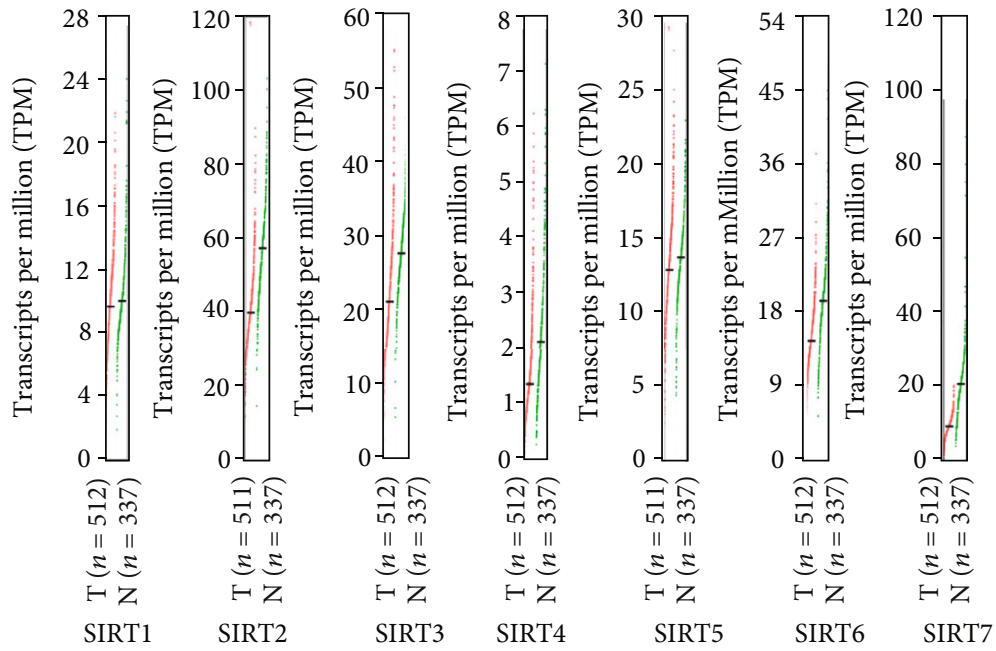

(a)
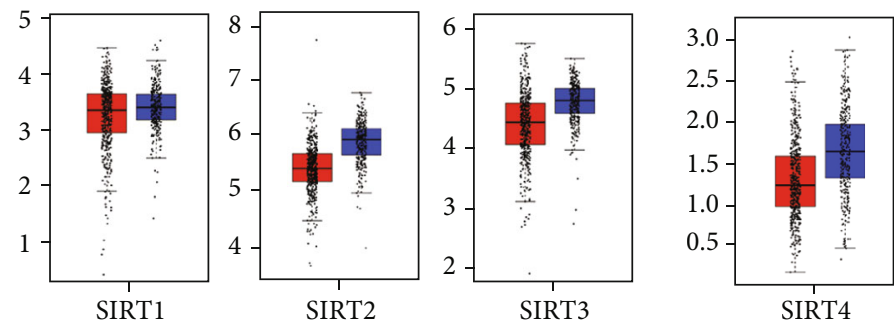

$\operatorname{num}(\mathrm{T})=512$;

num $(\mathrm{T})=512$;

num $(\mathrm{T})=512$;

$\operatorname{num}(\mathrm{N})=337$

num $(\mathrm{T})=512$;

$\operatorname{num}(\mathrm{N})=337$
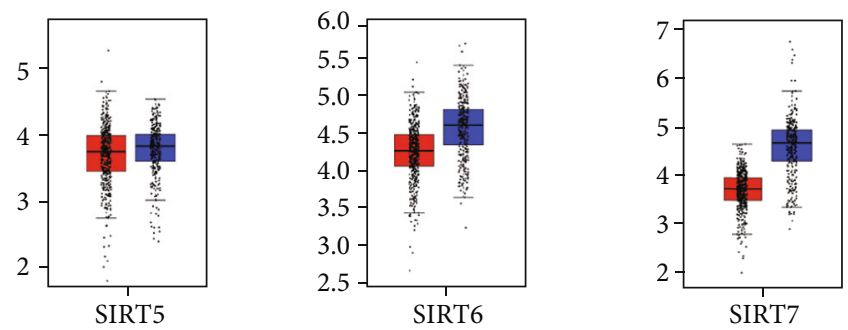

num $(\mathrm{T})=512$;

$\operatorname{num}(\mathrm{N})=337$

$$
\begin{aligned}
& \operatorname{num}(\mathrm{T})=512 ; \\
& \operatorname{num}(\mathrm{N})=337
\end{aligned}
$$

num $(T)=512$;

(b)

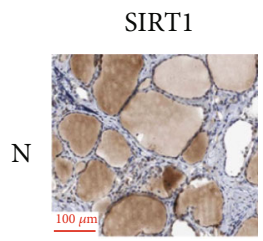

SIRT2
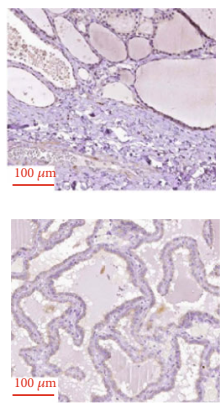

SIRT3
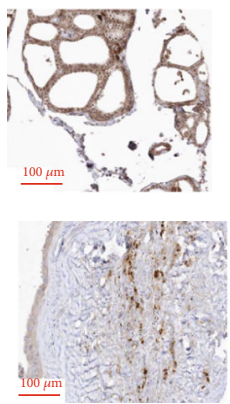

SIRT4
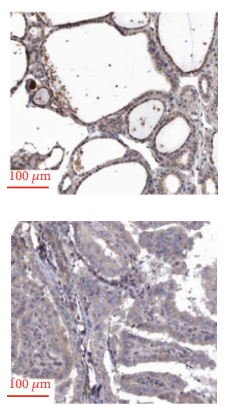

(c)
SIRT5
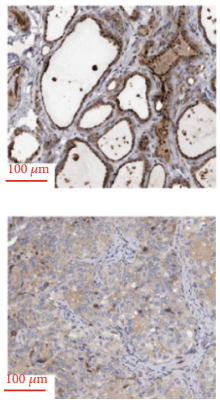

SIRT6
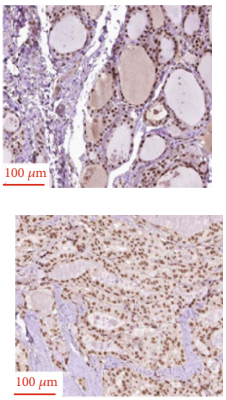

SIRT7
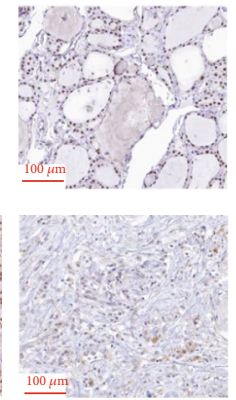

FIGURE 2: The expression of SIRT family members in DTC using GEPIA. (a) Scatter diagram showing the expression profile of SIRTs in DTC and normal samples. Red color represents the SIRT expression level in tumor samples, and green color represents the SIRT expression level in normal samples. (b) Box plot showing the expression of SIRTs in DTC and normal samples. Red color represents the SIRT expression level in tumor samples, and blue color represents the SIRT expression level in normal samples. (c) Representative IHC images of SIRTs in DTC. $\mathrm{T}$ represents tumor tissues and $\mathrm{N}$ represents normal tissues. Positive staining was mainly concentrated at the nucleus. 


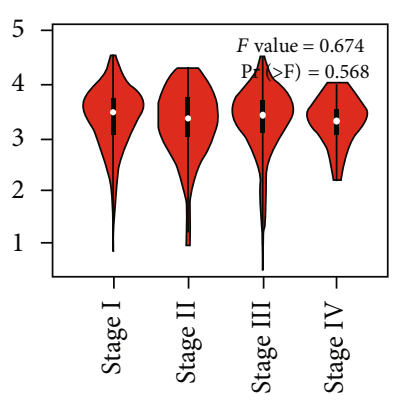

SIRT1

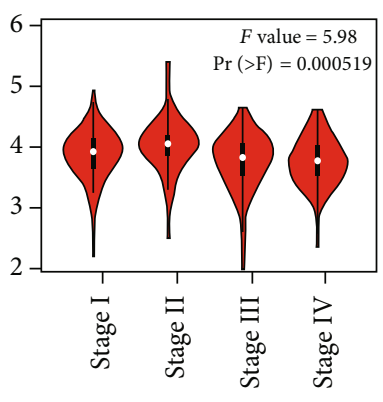

SIRT5

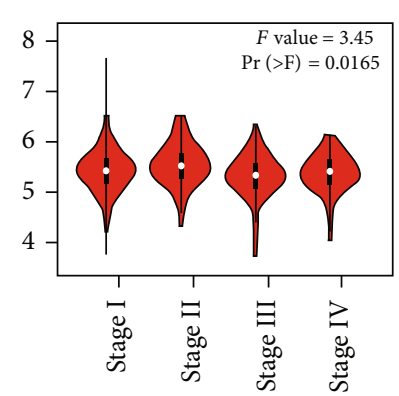

SIRT2

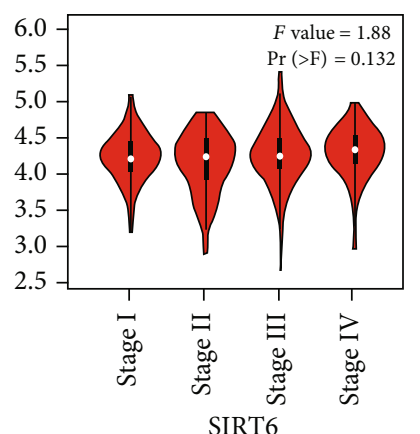

(a)

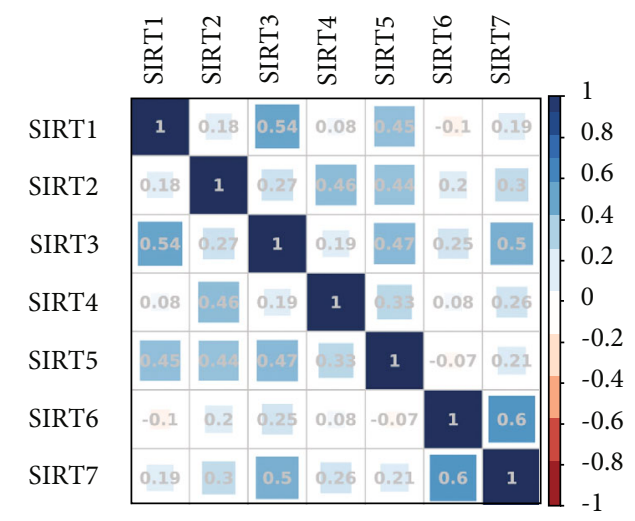

(b)

Figure 3: Continued.

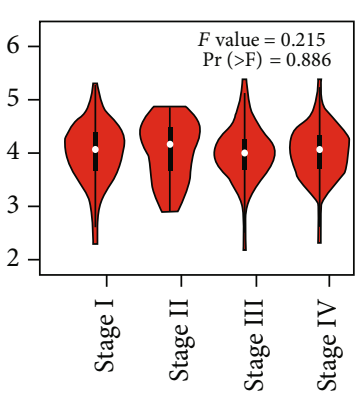

SIRT3

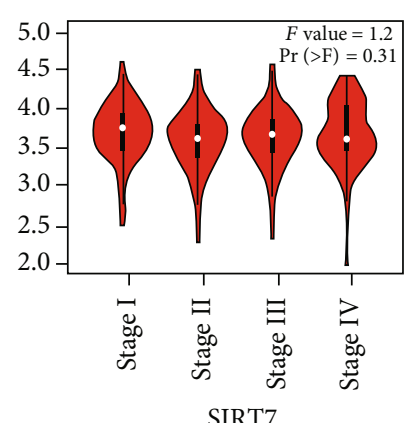

SIRT7

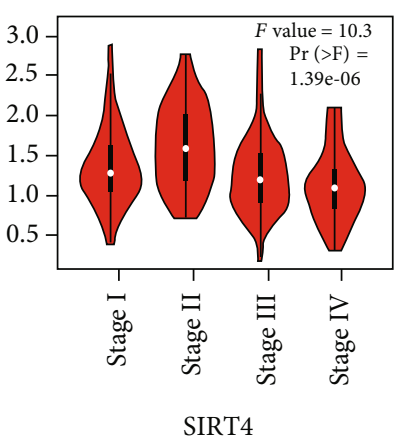

SIRT4 


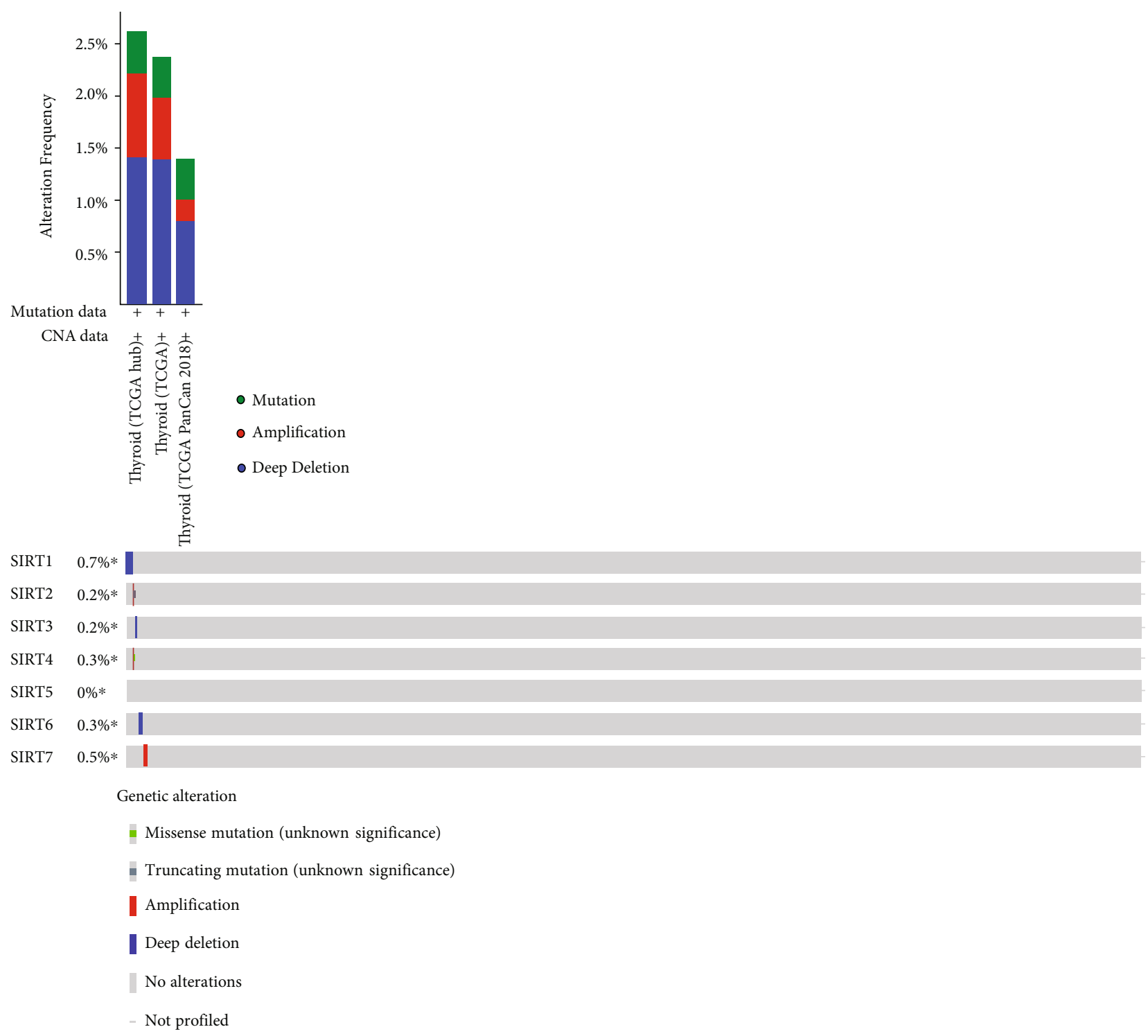

(c)

Figure 3: Correlation between SIRT expression and tumor stage in DTC (GEPIA) and genetic variation. (a) Association between the expression level of SIRTs and tumor stages. (b) Correlation analysis between SIRTs in DTC. Darker colors represent the higher correlation. (c) Genetic variation of SIRTs in DTC. Genetic variation included mutation, amplification, and deep deletion.

and SIRT6), truncating mutation (SIRT2), amplification (SIRT2, SIRT4, and SIRT7), and deep deletion (SIRT1, SIRT3, and SIRT6, Figure 3(c)). No alteration was recorded for SIRT5.

3.2. Prognosis Evaluation of SIRTs in DTC Patients. To explore the possible involvement of the SIRT family in the survival outcomes of DTC patients, the Kaplan-Meier Plotter tool was utilized to assess their survival rates by using the openly accessible DTC datasets. A positive correlation between the increased SIRT4 mRNA expression level with improved overall survival (OS) was recorded (Figure $4, p<$ $0.05)$. The increased SIRT7 mRNA expression had a trend toward better OS $(p=0.052)$. DTC patients with high SIRT2, SIRT3, SIRT4, and SIRT5 mRNA levels had longer diseasefree survival (DFS) time (Figure $4, p<0.001$ ).
3.3. Identification of Genes Correlated with SIRTs. With the aim of identifying the genes that were correlated with the expression of SIRTs, the Pearson correlation analysis was performed using the LinkedOmics database. The results indicated the expression of SIRTs was significantly correlated with a multitude of genes (Figures 5 and 6). We found that TNKS2, PHF3, MORC3, SMC3, and SPOPL were genes most positively correlated with SIRT1 while AP2S1, EXOSC4, GPS1, NDUFS8, and POLR2L were the genes most negatively associated with SIRT1 (Figures 5 and 6). Genes such as NAPA, BCAT2, and GNAS were the most positively correlated with SIRT2 while NOTCH2, AHNAK, ASAP2, and SGMS2 were genes most negatively associated with SIRT2 (Figures 5 and 6). Genes positively associated with SIRT3 were represented by DMAP1, TCEA2, MYST1, and SNRPA whereas the most negatively correlated genes were 

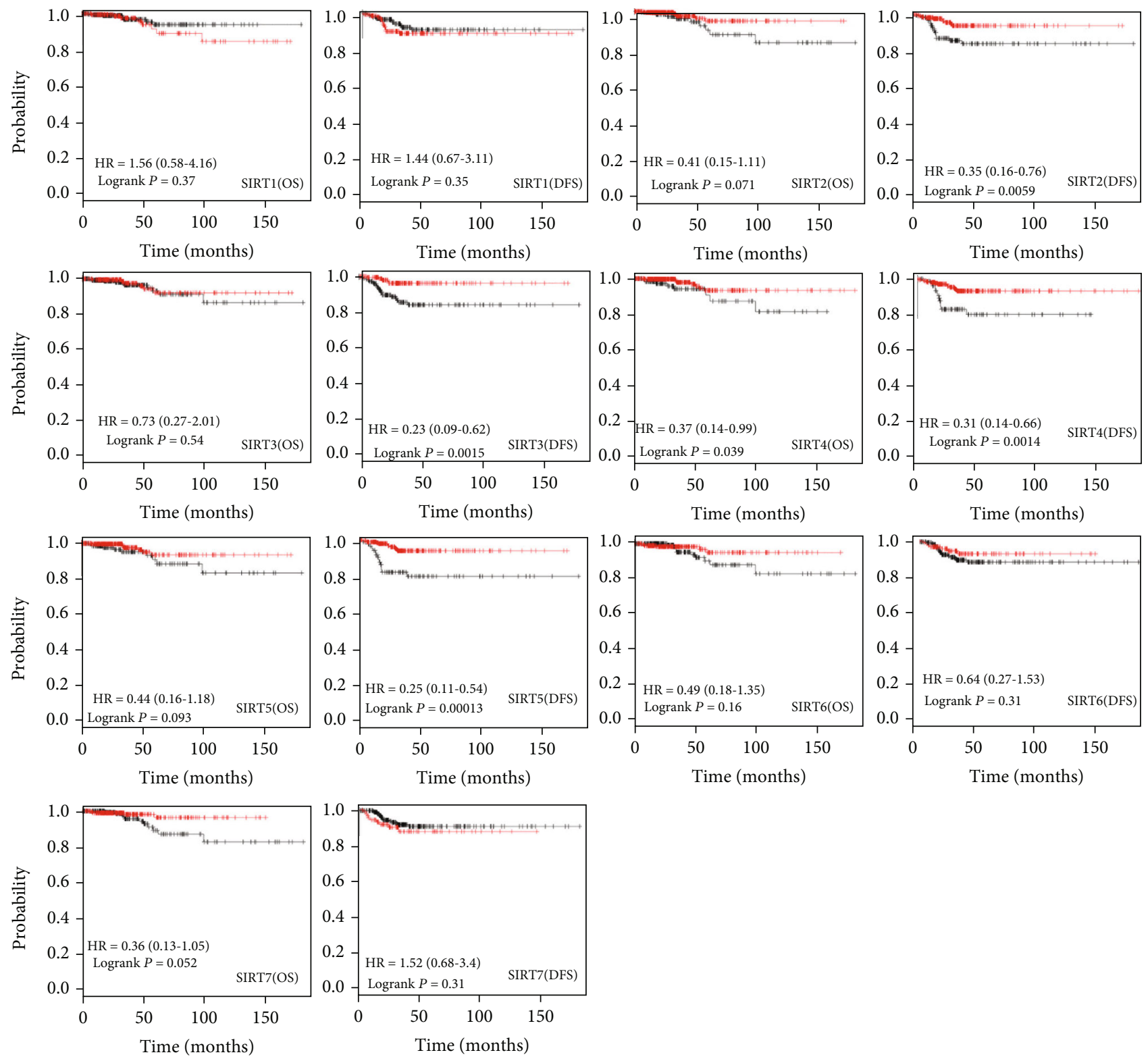

Expression

— Low

- High

FIGURE 4: The prognostic value of mRNA level of SIRT factors in DTC (Kaplan-Meier Plotter), including OS and DFS analysis. HR > 1.0 represents a risky gene, and $\mathrm{HR}<1.0$ represents a protective gene.

HEATR5A and YME1L1 (Figures 5 and 6). SLC25A42, ZNF346, and LOH12CR2 were genes most positively associated with SIRT5 while KCNQ3, FLNA, RUNX1, and FN1 were those most significantly and negatively associated with SIRT4 (Figures 5 and 6). The genes most positively correlated with SIRT5 were OXSM, SFXN4, COQ9, and NDUFA5 while those most negatively regulated with this gene were B4GALT5 and GALNT5 (Figures 5 and 6). SIRT6 was most positively associated with LSM7 and FKBP8 but most negatively associated with $S T T 3 B$ and CLCN3 while genes most positively associated with SIRT7 were SPSB3, ZGPAT, PUS1, and TSEN54 but PRKAR2A and PDZD8 were the most negatively associated with SIRT7 (Figures 5 and 6). Specially, BRAF mutation is the most common gene alteration in DTC [30]. We examined the correlation between SIRTs and BRAF and found that BRAF was positively associated with SIRT1, SIRT2, SIRT3, SIRT4, SIRT5, and 7 but negatively associated with SIRT6 $(p<0.05)$.

3.4. Functional Analysis of SIRTs. In order to uncover the functions prominently associated with the SIRTs and their coregulated genes, functional enrichment analysis was performed on a set of genes containing SIRTs and genes correlated with SIRTs with $r$ greater than 0.8 and $p<0.05$. The 

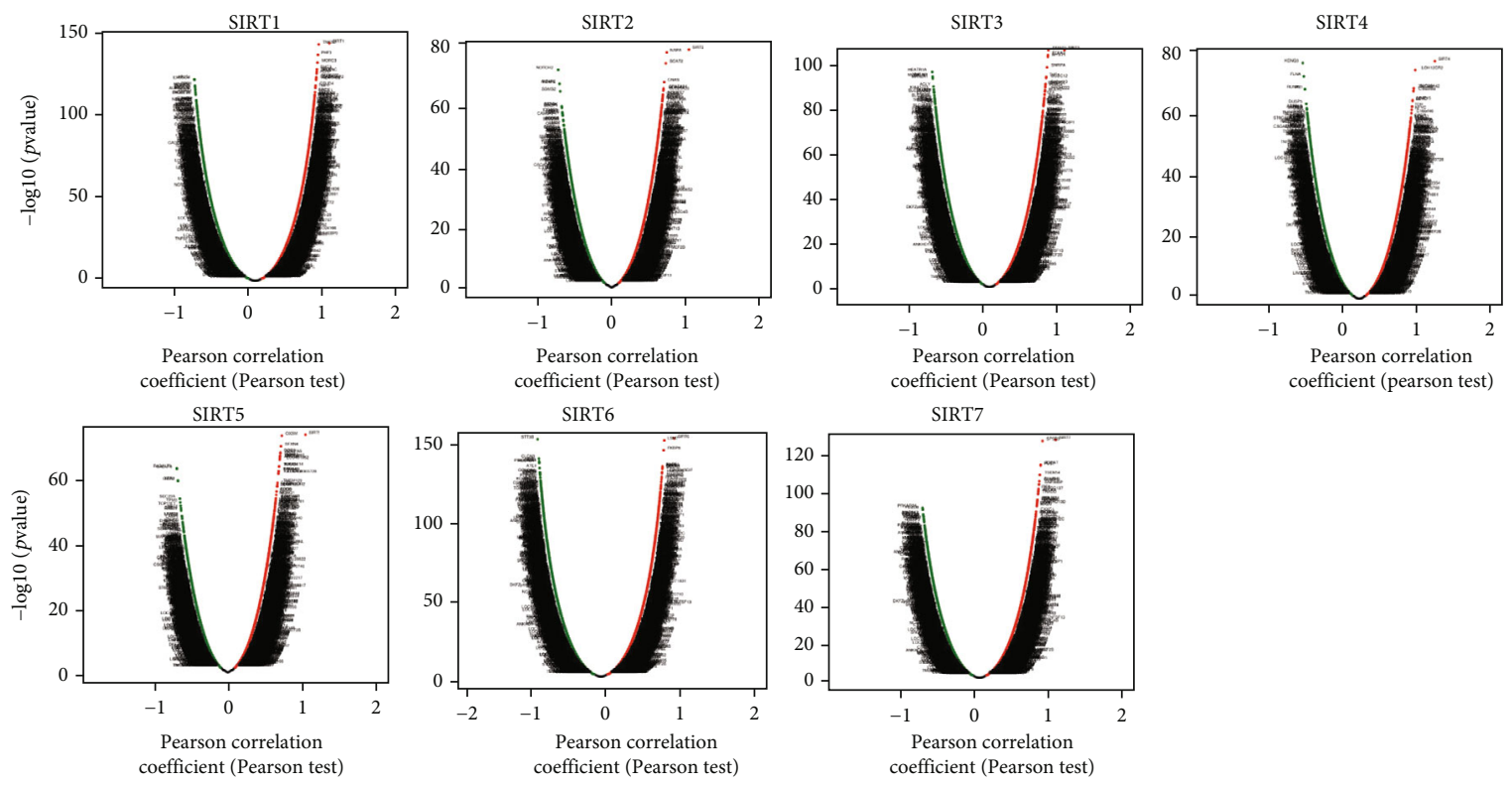

FIGURE 5: Volcano plot of association results showing the correlation of different SIRTs with gene expression in DTC (LinkedOmics). Red dots represent positive correlation, and green dots represent negative correlation.

results indicated that SIRTs and coregulated genes were involved in biological processes (BP) of protein deacetylation, peptidyl-lysine modification, protein ADP-ribosylation, and protein diacylation (Figure $7(\mathrm{a})$ ). In the category of cellular component (CC), cohesin complex, chromatin, and mitotic spindle pole were the most represented gene ontology (GO) terms while in the category of molecular function (MF), $\mathrm{NAD}+$ binding, NAD-dependent protein deacetylase activity, and protein deacetylase activity were the predominant GO terms (Figures 7(b) and 7(c)). The KEGG pathway enrichment analysis showed that nicotinamide and nicotinamide metabolism, basal transcription factors, central carbon metabolism in cancer, Huntington's disease, and FOXO signaling pathway were the most significantly enriched signaling pathways associated with SIRTs and their correlated genes in DTC (Figure $7(\mathrm{~d})$ ). In the pathway of central carbon metabolism in cancer, SIRT6 could directly inhibit hypoxia-inducible factor $1 \alpha(H I F-1 \alpha)$, and SIRT3 could inhibit HIF-1 $\alpha$ by repressing HIF-1 signaling, which then affected metabolic process such as glycolysis and tricarboxylic acid (TCA) cycle (Figure 8 ). The Ras/Raf/ERK/MAPK pathway was also regulated by SIRT6, thus contributing to $c-M y c$ deregulated expression. In addition, SIRT1, together with BARF, could modulate the FOXO signaling pathway (Figure 9).

\section{Discussion}

Dysregulation of SIRTs has been investigated in diverse cancers [24]. Although the function and prognostic values of SIRTs had been partly validated in various cancers, no bioinformatics analysis of SIRTs has been performed in DTC [31, 32]. This study reported for the first time the mRNA expression profiles and clinical value of SIRTs in DTC. Our findings will help leverage existing knowledge to improve treatment design and improve the prognosis of patients with DTC.
SIRT1 was generally known as an oncogene and involved in multiple cellular processes including cell cycle, apoptosis, and cancer metastasis [33]. SIRT1 was acknowledged as a direct downstream target of $m i R-212$, which hindered the proliferation and promoted the apoptosis of thyroid cancer cells by negatively regulating SIRT1 [34]. It has also been reported that SIRT1-Foxp3 signaling-mediated regulation of Bax and p21 mRNA expression is a hallmark molecular event in DTC and shows significant resistance to genotoxic stress induced by the chemotherapeutic agent etoposide [22]. Li et al. [35] discovered that SIRT7 could promote tumorigenesis by acting on the DBC1/SIRT1 axis in PTC cells. The result was consistent with our findings that SIRT1 and SIRT7 were correlated significantly $(r=0.19, p<0.001)$. Roehlen et al. [36] demonstrated that the vitamin D-SIRT1FOXO3a axis played a pivotal role in DTC and Hashimoto thyroiditis. Our KEGG results also showed SIRT1 affected the FOXO signaling pathway along with BRAF. In the study, however, no significant associations were found between SIRT1 and clinical characteristics.

So far, our knowledge on the expression and regulation of SIRT2 in DTC is limited. In contrast to previous results suggesting a broad tumor-promoting effect for SIRT2 [37], our study indicated that SIRT2 expression was decreased in DTC tissues compared to the nontumorous tissues. Its expression pattern was significantly associated with tumor stage. Higher expression of SIRT2 was not significantly associated with OS but was correlated with improved DFS in all patients with DTC, which suggested SIRT2 as a possible target of treatment.

SIRT3, a nicotinamide adenine dinucleotide- (NAD-) dependent deacetylase, was often recognized as a tumorsuppressor gene [38]. However, SIRT3 was reported to be highly expressed in DTC compared to benign thyroid tumor and might involve mitochondrial alterations [39]. 

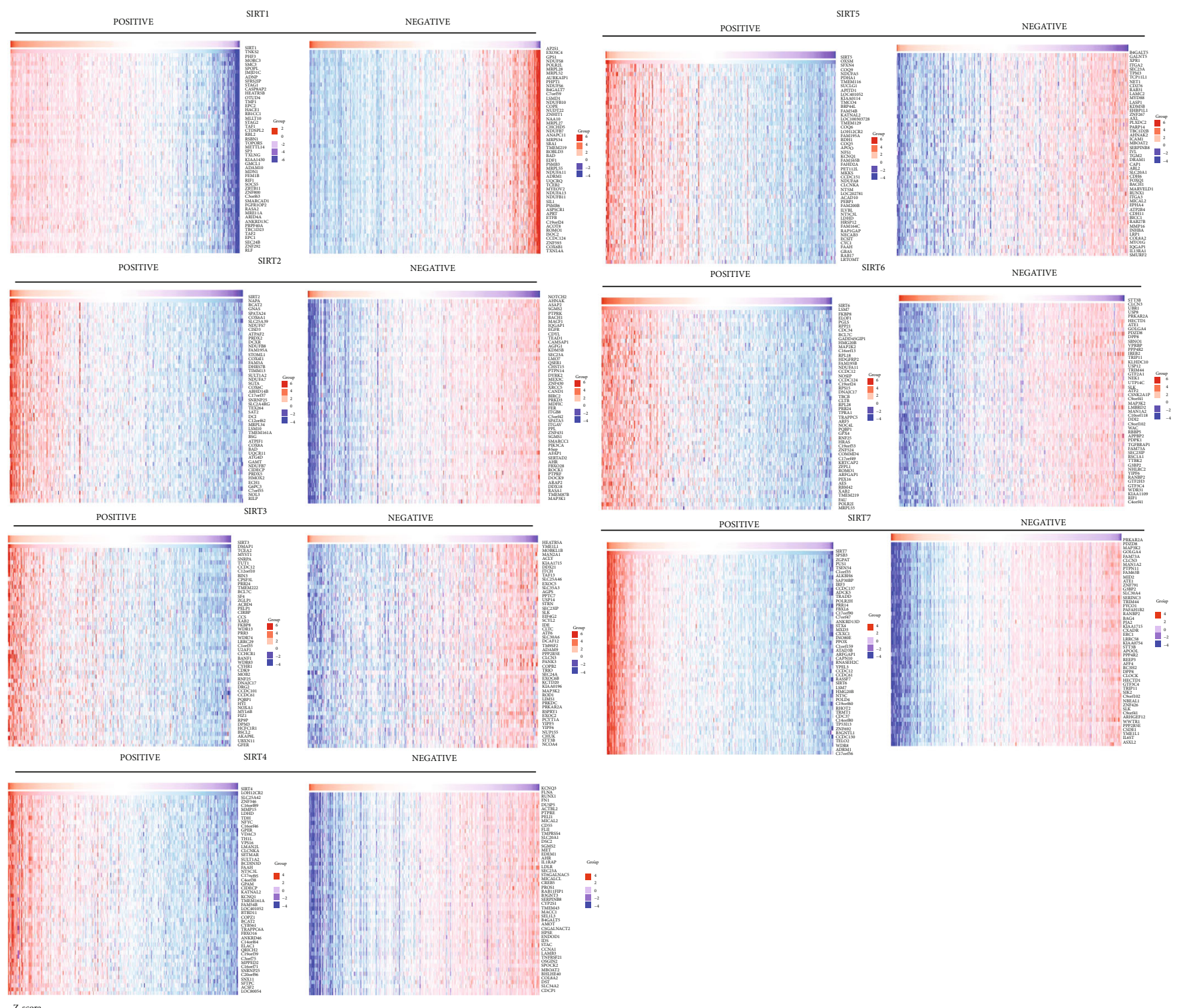

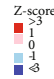

FIgURE 6: Heatmap plot of association results showing the correlation of different SIRTs with gene expression in DTC (LinkedOmics). Top 50 genes with the greatest correlation were presented.

Wang et al. showed that $m i R-1225-5 p$ could promote DTC cell proliferation and metastasis via targeting SIRT3 directly [40]. These results and ours were not in accordance. Our findings indicated that the mRNA expression level of SIRT3 was slightly lower in DTC tissue than in normal tissue, and patients with high DTC expression had better DFS. Additionally, there have been a few studies on SIRT3/HIF-1 $\alpha$ pathway in cervical cancer and hepatocellular cancer but not in DTC [41-43]. More validation studies need to be performed to further investigate the role of SIRT3 in DTC.

SIRT4 displayed deacetylase activity and were involved in regulating cellular energy metabolism [44]. Studies showed that SIRT4 was also reported to significantly decrease in thyroid cancer and inhibit glutamine metabolism and thus inhibit cell proliferation and invasion [45]. This is consistent with our predictions. In our study, we demonstrated that the expression of SIRT4 in DTC tissues was downregulated, and its expression was associated with tumor progression. Moreover, low expression of SIRT4 was markedly correlated with poor OS and DFS, which corroborated with the findings that SIRT4 is an antitumor gene [46]. This suggests that SIRT4 may be considered a potential biomarker of poor prognosis and an effective molecular target of treatment for DTC.

SIRT5 played a very important role in fatty acid oxidation, glycolysis, TCA cycle, apoptosis, and antioxidant defense [47]. Some researchers also found that SIRT5 was upregulated in cisplatin-resistant ovarian cancer cells compared with cisplatin-sensitive cells [48]. In addition, SIRT5 was found to promote cisplatin resistance (HO-1) pathway in ovarian cancer by modulating Nrf2/heme oxygenase 1 


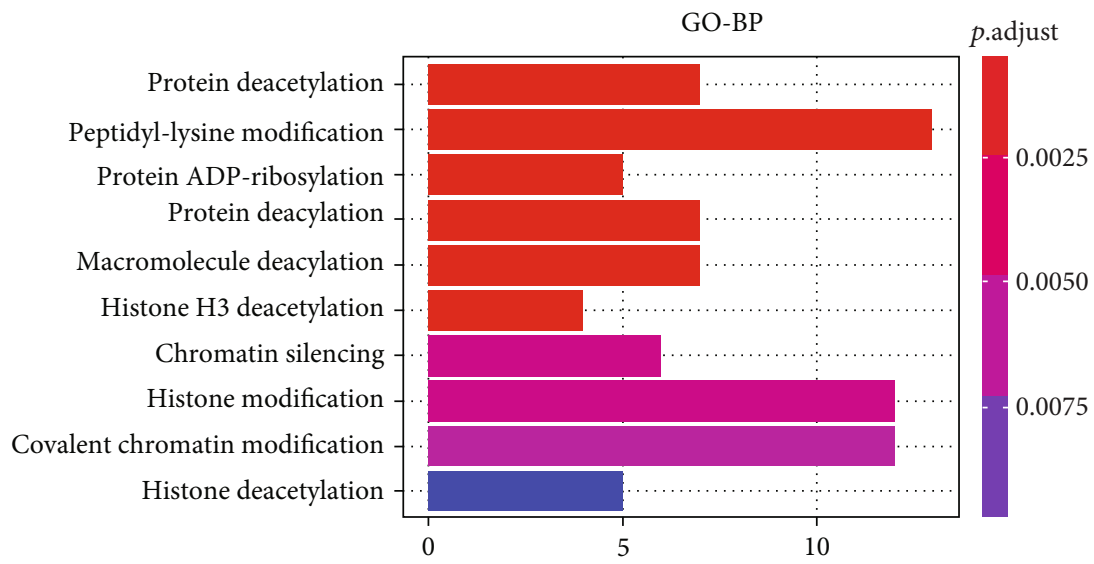

(a)

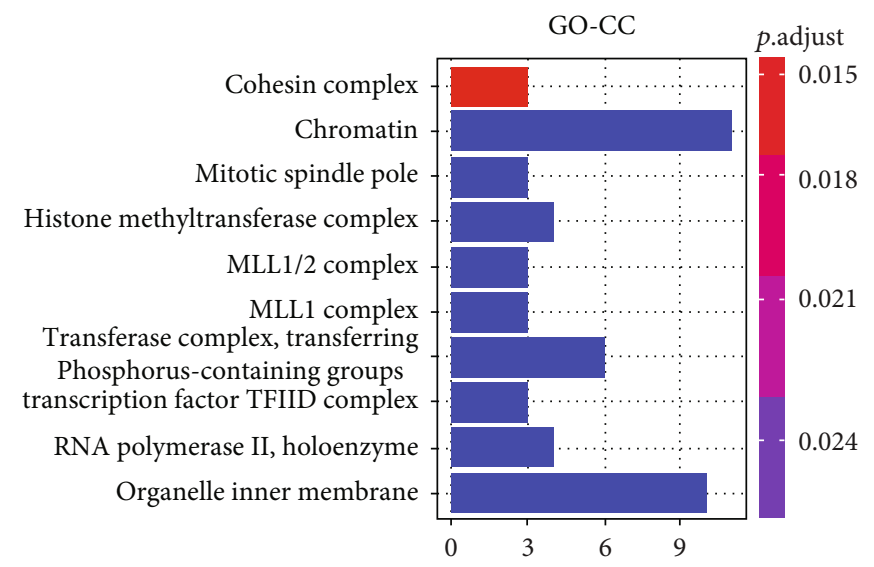

(b)

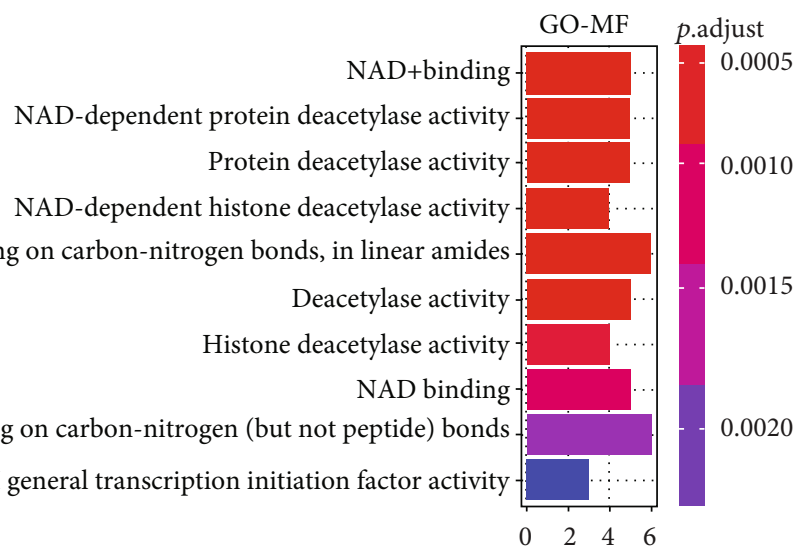

(c)

Figure 7: Continued. 


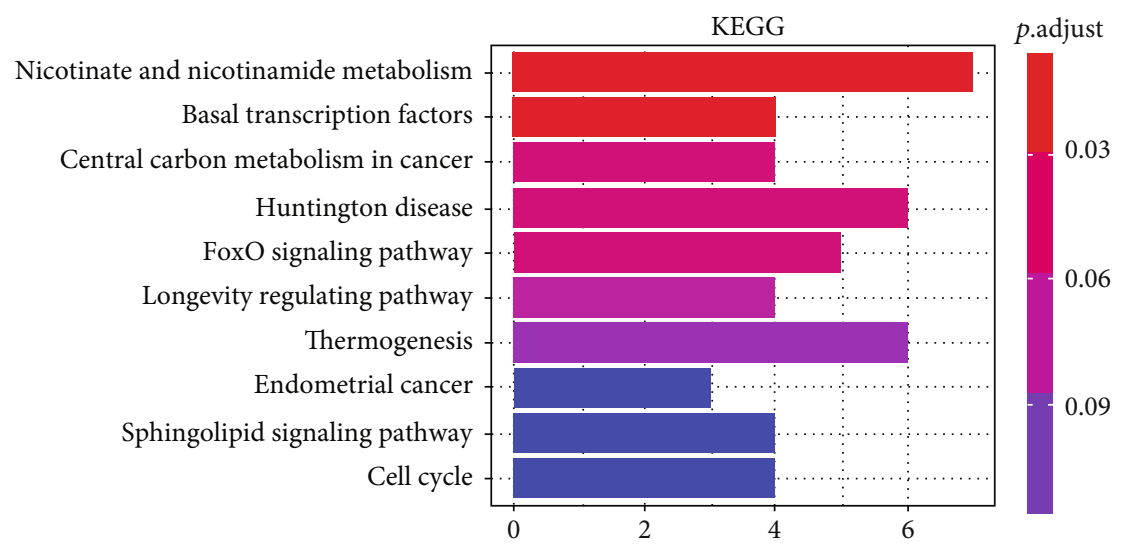

(d)

FIGURE 7: Functional enrichment analysis of SIRTs and coregulated genes in DTC. (a) Biological process. (b) Cellular component. (c) Molecular function. (d) KEGG pathways. The intensity of the colors represents the $p$ value (the redder the color, the lower $p$ value). Top 10 significant terms were presented.

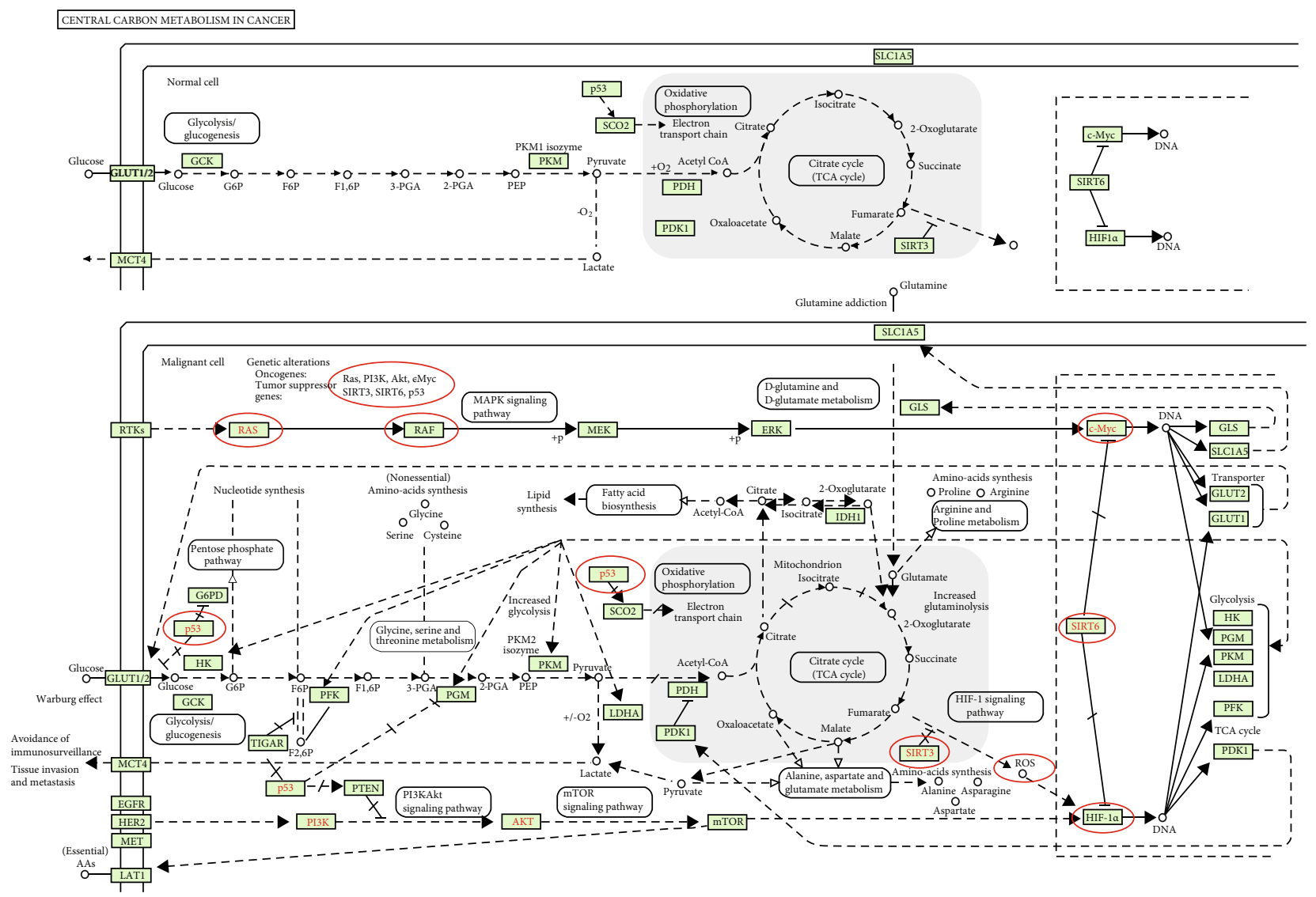

FIGURE 8: Carbon metabolism in cancer pathway. SIRTs and coregulated genes are noted in a red circle. Solid arrow represents epicardial activation; dashed arrow represents septal activation; T-shaped arrow represents inhibition.

axis [48]. However, no study has reported the role of SIRT5 in DTC. In the present study, low expression of SIRT5 was observed in DTC patients with advanced diseases and significantly correlated with improved DFS, suggesting SIRT5 may function as a tumor-suppressor gene.

Previous works indicated that SIRT6 plays a relevant role in aging biochemical functions involved in tumor progres- sion and could constitute an antitumor therapeutic target [49]. Qu et al. [50] demonstrated that SIRT6 enhanced their malignant behavior through the $B R A F / E R K / M c l-1$ pathway. Our studies also found the significant relationship between SIRT6 and BRAF (Figures 3(b) and 8). Nevertheless, SIRT6 seemed to have no effect on tumor stage or clinical outcomes. 


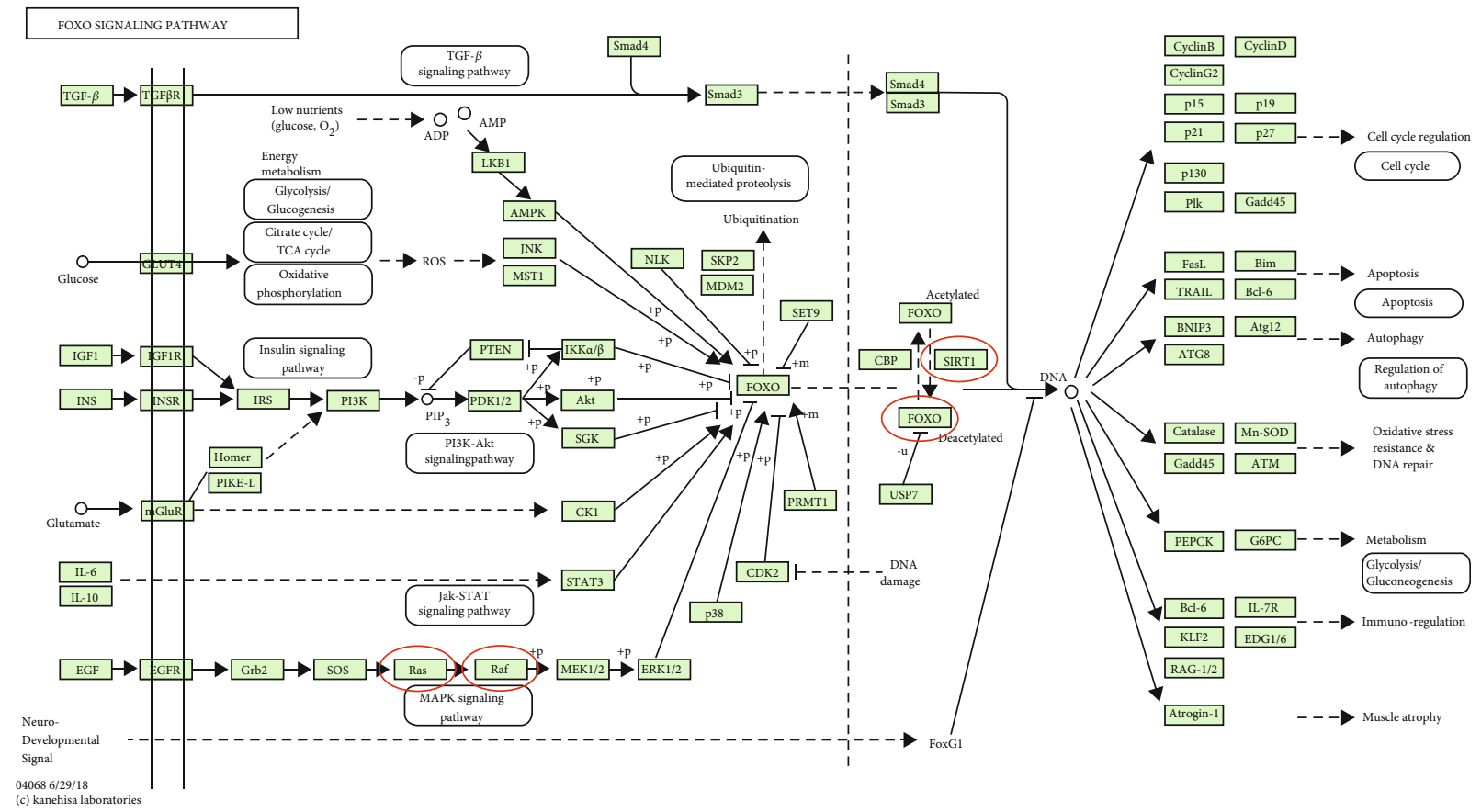

FIgURE 9: The FOXO signaling pathway. SIRTs and coregulated genes are noted in a red circle. Solid arrow represents epicardial activation; dashed arrow represents septal activation; T-shaped arrow represents inhibition.

SIRT7 was upregulated in multiple cancers including DTC and could promote the tumorigenesis of DTC cells in vitro and in vivo $[35,51-53]$. SIRT7 is reported to be correlated with active rRNA genes (rDNA) and actively increases outgrowth and proliferation of U2OS cells [54]. Compared to the SIRT7-wildtype hepatoma cell line, the SIRT7-deficient cell line exhibited exquisite sensitivity to doxorubicin via the SIRT7-P53-NOXA axis [55]. Inhibition of SIRT7 using small interfering RNAs inhibits tumor resistance to radiation $[56,57]$. In the present study, SIRT7 expression was not associated with tumor stage and prognosis in DTC.

Functional enrichment of SIRTs and their coregulated genes in DTC indicated that these genes were involved in protein deacetylation, peptidyl-lysine modification, protein ADP-ribosylation, and protein diacylation. The most significant pathways were nicotinamide and nicotinamide metabolism pathways and basal transcription factors. These results indicated that SIRTs may participate in the pathogenesis of DTC by regulating these pathways and biological processes. In NIH3T3 cells, SIRT1 causes ubiquitination and degradation of FOXO3, a FOXO transcription factor family member that can play a crucial role in tumor suppression and metabolism and may act as oncogene [58]. Previous studies reported that SIRT2 mediates the acetylation of pyruvate kinase to regulate tumor growth [59]. SIRT3, downregulated in cholangiocarcinoma (CCA) patients, can prevent tumor progression by inhibiting the HIF1 $\alpha / P D K 1 / P D H A 1$ pathway [60]. SIRT5 was also found to modulate the deacetylation of $L D H B$ and induce the autophagy in colorectal cancer [61]. Previous study showed that SIRT6 might act as antioncogenesis factor by inhibiting $H I F-1 \alpha$, an angiogenesis-promoting mol- ecule, in lung cancer [62], and by inhibiting $c-M y c$ gene and ribosome biosynthesis [63]. Since our results showed decreased expression levels of SIRT3 and SIRT6 in DTC tissues, we estimate that SIRT3 and SIRT6 take effect through metabolic processes such as glycolysis and TCA cycle. Similarly, SIRT7 was found to counteract cancer development by the deacetylation of WDR77 [64]. These studies support our finding that the SIRTs and coregulated genes were involved in deacetylation in DTC. Moreover, our study corroborated with previous studies demonstrating that nicotinamide metabolism regulates cancer processes $[65,66]$.

\section{Conclusion}

In summary, we performed a systematic exploration to examine the expression profiles and clinical value of the SIRT family proteins in DTC and have provided an overview of these SIRTs in DTC. Our findings suggest that high expression of SIRT2, SIRT3, SIRT4, SIRT5, and SIRT6 in DTC may indicate that they have significant regulatory functions in thyroid carcinogenesis. Therefore, SIRT2, SIRT3, SIRT4, SIRT5, and SIRT6 may be relevant therapeutic targets for DTC. Moreover, the expression of SIRT2, SIRT3, SIRT4, and SIRT5 may have potential as prognostic markers for determining the survival and prognosis of DTC.

\section{Data Availability}

The datasets used in the present study can be obtained in The Cancer Genome Atlas (https://portal.gdc.cancer.gov/). 


\section{Conflicts of Interest}

The authors declare that there is no conflict of interest regarding the publication of this paper.

\section{Authors' Contributions}

YW designed the study, analyzed the data, and wrote the manuscript. LY performed the experiments, revised the manuscript, and assisted in the study design. All the authors read and approved the final manuscript.

\section{References}

[1] M. Xing, "Molecular pathogenesis and mechanisms of thyroid cancer," Nature Reviews Cancer, vol. 13, no. 3, pp. 184-199, 2013.

[2] M. I. Abdullah, S. M. Junit, K. L. Ng, J. J. Jayapalan, B. Karikalan, and O. H. Hashim, "Papillary thyroid cancer: genetic alterations and molecular biomarker investigations," International Journal of Medical Sciences, vol. 16, no. 3, pp. 450-460, 2019.

[3] D. Rusinek, E. Chmielik, J. Krajewska et al., "Current advances in thyroid cancer management. Are we ready for the epidemic rise of diagnoses?," International Journal of Molecular Sciences, vol. 18, no. 8, article 1817, 2017.

[4] L. Valerio, L. Pieruzzi, C. Giani et al., "Targeted therapy in thyroid cancer: state of the art," Clinical Oncology (Royal College of Radiologists), vol. 29, no. 5, pp. 316-324, 2017.

[5] D. Viola, L. Valerio, E. Molinaro et al., "Treatment of advanced thyroid cancer with targeted therapies: ten years of experience," Endocrine-Related Cancer, vol. 23, no. 4, pp. R185R205, 2016.

[6] D. F. Schneider and H. Chen, "New developments in the diagnosis and treatment of thyroid cancer," CA: a Cancer Journal for Clinicians, vol. 63, no. 6, pp. 373-394, 2013.

[7] V. Tiedje, D. Zwanziger, S. Ting, K. W. Schmid, and D. Fuhrer, "Molecular pathogenesis of thyroid tumors," Deutsche Medizinische Wochenschrift, vol. 140, no. 8, pp. 578-582, 2015.

[8] M. Melo, A. Gaspar da Rocha, R. Batista et al., "TERT, BRAF, and NRAS in primary thyroid cancer and metastatic disease," The Journal of Clinical Endocrinology and Metabolism, vol. 102, no. 6, pp. 1898-1907, 2017.

[9] M. Zou, E. Y. Baitei, A. S. Alzahrani et al., "Concomitant RAS, RET/PTC, or BRAF mutations in advanced stage of papillary thyroid carcinoma," Thyroid, vol. 24, no. 8, pp. 1256-1266, 2014.

[10] H. G. Vuong, T. Kondo, N. Oishi et al., "Paediatric follicular thyroid carcinoma - indolent cancer with low prevalence of RAS mutations and absence of PAX8-PPARG fusion in a Japanese population," Histopathology, vol. 71, no. 5, pp. 760-768, 2017.

[11] R. Nagy, S. Ganapathi, I. Comeras et al., "Frequency of germline PTEN mutations in differentiated thyroid cancer," Thyroid : Official Journal of the American Thyroid Association, vol. 21, no. 5, pp. 505-510, 2011.

[12] A. S. Alzahrani, A. K. Murugan, E. Qasem, M. Alswailem, H. Al-Hindi, and Y. Shi, "Single point mutations in pediatric pifferentiated thyroid cancer," Thyroid, vol. 27, no. 2, pp. 189-196, 2017.
[13] S. K. Yoo, S. Lee, S. J. Kim et al., "Comprehensive analysis of the transcriptional and mutational landscape of follicular and papillary thyroid cancers," PLoS Genetics, vol. 12, no. 8, article e1006239, 2016.

[14] A. Vassilopoulos, K. S. Fritz, D. R. Petersen, and D. Gius, "The human sirtuin family: evolutionary divergences and functions," Human Genomics, vol. 5, no. 5, pp. 485-496, 2011.

[15] J. M. Beauharnois, B. E. Bolivar, and J. T. Welch, "Sirtuin 6: a review of biological effects and potential therapeutic properties," Molecular BioSystems, vol. 9, no. 7, pp. 1789-1806, 2013.

[16] C. Cantó, A. A. Sauve, and P. Bai, "Crosstalk between poly(ADP-ribose) polymerase and sirtuin enzymes," Molecular Aspects of Medicine, vol. 34, no. 6, pp. 1168-1201, 2013.

[17] A. A. Sag, F. Selcukbiricik, and N. M. Mandel, "Evidence-based medical oncology and interventional radiology paradigms for liver-dominant colorectal cancer metastases," World Journal of Gastroenterology, vol. 22, no. 11, pp. 3127-3149, 2016.

[18] J. D. Lovaas, L. Zhu, C. Y. Chiao, V. Byles, D. V. Faller, and Y. Dai, "SIRT1 enhances matrix metalloproteinase-2 expression and tumor cell invasion in prostate cancer cells," The Prostate, vol. 73, no. 5, pp. 522-530, 2013.

[19] D. H. Shin, Y.-J. Choi, P. Jin et al., "Distinct effects of SIRT1 in cancer and stromal cells on tumor promotion," Oncotarget, vol. 7, no. 17, pp. 23975-23987, 2016.

[20] C. K. Singh, G. Chhabra, M. A. Ndiaye, L. M. Garcia-Peterson, N. J. Mack, and N. Ahmad, "The role of sirtuins in antioxidant and redox signaling," Antioxidants \& Redox Signaling, vol. 28, no. 8, pp. 643-661, 2018.

[21] A. E. Wyman and S. P. Atamas, "Sirtuins and accelerated aging in scleroderma," Current Rheumatology Reports, vol. 20, no. 4, pp. 16-16, 2018.

[22] K. H. Kweon, C. R. Lee, S. J. Jung et al., "Sirt1 induction confers resistance to etoposide-induced genotoxic apoptosis in thyroid cancers," International Journal of Oncology, vol. 45, no. 5, pp. 2065-2075, 2014.

[23] S. Paredes, L. Villanova, and K. F. Chua, "Molecular pathways: emerging roles of mammalian sirtuin SIRT7 in cancer," Clinical Cancer Research : An Official Journal of The American Association for Cancer Research, vol. 20, no. 7, pp. 17411746, 2014.

[24] M. Roth and W. Y. Chen, "Sorting out functions of sirtuins in cancer," Oncogene, vol. 33, no. 13, pp. 1609-1620, 2014.

[25] W. Feng, L. Zhen, R. Z. Luo et al., "The ARHI tumor suppressor gene is repressed by multiple histone deacetylases (HDACs) in breast cancer cells.," Cancer Research, vol. 65, pp. 643-643, 2005.

[26] P. Behzadi, E. Behzadi, and S. M. Alavian, "DNA microarray technology in HBV genotyping," Minerva Medica, vol. 108, no. 5, pp. 473-476, 2017.

[27] T. Li, C. Mo, X. Qin, S. Li, Y. Liu, and Z. Liu, "Glycoprofiling of early gastric cancer using lectin microarray technology," Clinical Laboratory, vol. 64, no. 1, pp. 153-161, 2018.

[28] L. Lamartina, G. Grani, C. Durante, and S. Filetti, "Recent advances in managing differentiated thyroid cancer," F1000Research, vol. 7, pp. 86-86, 2018.

[29] S. V. Vasaikar, P. Straub, J. Wang, and B. Zhang, "LinkedOmics: analyzing multi-omics data within and across 32 cancer types," Nucleic Acids Research, vol. 46, no. D1, pp. D956D963, 2018.

[30] Y. Li, Y. Zhang, S. Xiao et al., "Mps1 is associated with the BRAF(V600E) mutation but does not rely on the classic 
RAS/RAF/MEK/ERK signaling pathway in thyroid carcinoma," Oncology Letters, vol. 15, no. 6, pp. 9978-9986, 2018.

[31] S. M. Jeong and M. C. Haigis, "Sirtuins in cancer: a balancing act between genome sability and metabolism," Molecules and Cells, vol. 38, no. 9, pp. 750-758, 2015.

[32] Y. I. Cha and H. S. Kim, "Emerging role of sirtuins on tumorigenesis: possible link between aging and cancer," $B M B$ Reports, vol. 46, no. 9, pp. 429-438, 2013.

[33] C. X. Deng, "SIRT1, is it a tumor promoter or tumor suppressor?," International Journal of Biological Sciences, vol. 5, no. 2, pp. 147-152, 2009.

[34] D. Li, L. Bai, T. Wang et al., "Function of miR-212 as a tumor suppressor in thyroid cancer by targeting SIRT1," Oncology Reports, vol. 39, no. 2, pp. 695-702, 2018.

[35] H. Li, Z. Tian, Y. Qu et al., "SIRT7 promotes thyroid tumorigenesis through phosphorylation and activation of Akt and p70S6K1 via DBC1/SIRT1 axis," Oncogene, vol. 38, no. 3, pp. 345-359, 2019.

[36] N. Roehlen, C. Doering, M. L. Hansmann et al., "Vitamin D, FOXO3a, and sirtuin 1 in Hashimoto's thyroiditis and differentiated thyroid cancer," Front Endocrinol (Lausanne), vol. 9, p. 527, 2018.

[37] N. A. Spiegelman, J. Y. Hong, J. Hu et al., "A small-molecule SIRT2 inhibitor that promotes K-Ras4a lsine ftty-acylation," ChemMedChem, vol. 14, no. 7, pp. 744-748, 2019.

[38] T. Y. Alhazzazi, P. Kamarajan, E. Verdin, and Y. L. Kapila, "SIRT3 and cancer: tumor promoter or suppressor?," Biochimica et Biophysica Acta, vol. 1816, no. 1, pp. 80-88, 2011.

[39] R. Shackelford, S. Hirsh, K. Henry, A. Abdel-Mageed, E. Kandil, and D. Coppola, "Nicotinamide phosphoribosyltransferase and SIRT3 expression are increased in welldifferentiated thyroid carcinomas," Anticancer Research, vol. 33, no. 8, pp. 3047-3052, 2013.

[40] S. Wang, X. Chen, Z. Zhang, and Z. Wu, "MicroRNA-1225-5p inhibits the development and progression of thyroid cancer via targeting sirtuin 3," Pharmazie, vol. 74, no. 7, pp. 423-427, 2019.

[41] X. L. Zhao and C. Z. Yu, "Vosaroxin induces mitochondrial dysfunction and apoptosis in cervical cancer HeLa cells: involvement of AMPK/Sirt3/HIF-1 pathway," Chemico-Biological Interactions, vol. 290, pp. 57-63, 2018.

[42] S. de Matteis, E. Scarpi, A. M. Granato et al., "Role of SIRT-3, p-mTOR and HIF- $1 \alpha$ in hepatocellular carcinoma patients affected by metabolic dysfunctions and in chronic treatment with metformin," International Journal of Molecular Sciences, vol. 20, no. 6, p. 1503, 2019.

[43] P. T. Schumacker, "SIRT3 controls cancer metabolic reprogramming by regulating ROS and HIF," Cancer Cell, vol. 19, no. 3, pp. 299-300, 2011.

[44] S. M. Jeong, C. Xiao, L. W. Finley et al., "SIRT4 has tumorsuppressive activity and regulates the cellular metabolic response to DNA damage by inhibiting mitochondrial glutamine metabolism," Cancer Cell, vol. 23, no. 4, pp. 450-463, 2013.

[45] Z. Chen, J. Lin, S. Feng et al., "SIRT4 inhibits the proliferation, migration, and invasion abilities of thyroid cancer cells by inhibiting glutamine metabolism," Oncotargets and Therapy, vol. 12, pp. 2397-2408, 2019.

[46] Y. Tao, S. Yu, M. Chao, Y. Wang, J. Xiong, and H. Lai, "SIRT4 suppresses the PI3K/Akt/NFkappaB signaling pathway and attenuates HUVEC injury induced by oxLDL," Molecular Medicine Reports, vol. 19, no. 6, pp. 4973-4979, 2019.

[47] L. R. Bringman-Rodenbarger, A. H. Guo, C. A. Lyssiotis, and D. B. Lombard, "Emerging roles for SIRT5 in metabolism and cancer," Antioxidants \& Redox Signaling, vol. 28, no. 8, pp. 677-690, 2018.

[48] X. Sun, S. Wang, J. Gai et al., "SIRT5 promotes cisplatin resistance in ovarian cancer by suppressing DNA damage in a ROS-dependent manner via regulation of the $\mathrm{Nrf} 2 / \mathrm{HO}-1$ pathway," Frontiers in Oncology, vol. 9, pp. 754-754, 2019.

[49] L. Tasselli, W. Zheng, and K. F. Chua, "SIRT6: novel mechanisms and links to aging and disease," Trends in Endocrinology and Metabolism, vol. 28, no. 3, pp. 168-185, 2017.

[50] N. Qu, J. Q. Hu, L. Liu et al., "SIRT6 is upregulated and associated with cancer aggressiveness in papillary thyroid cancer via BRAF/ERK/Mcl1 pathway," International Journal of Oncology, vol. 50, no. 5, pp. 1683-1692, 2017.

[51] S. Zhang, P. Chen, Z. Huang et al., "Sirt7 promotes gastric cancer growth and inhibits apoptosis by epigenetically inhibiting miR-34a," Scientific Report, vol. 5, no. 1, article 9787, pp. 1-19, 2015.

[52] N. Ashraf, S. Zino, A. Macintyre et al., "Altered sirtuin expression is associated with node-positive breast cancer," British Journal of Cancer, vol. 95, no. 8, pp. 1056-1061, 2006.

[53] J. K. Kim, J. H. Noh, K. H. Jung et al., "Sirtuin7 oncogenic potential in human hepatocellular carcinoma and its regulation by the tumor suppressors MiR-125a-5p and MiR-125b," Hepatology, vol. 57, no. 3, pp. 1055-1067, 2013.

[54] E. Ford, R. Voit, G. Liszt, C. Magin, I. Grummt, and L. Guarente, "Mammalian Sir2 homolog SIRT7 is an activator of RNA polymerase I transcription," Genes \& Development, vol. 20, no. 9, pp. 1075-1080, 2006.

[55] J. Zhao, A. Wozniak, A. Adams et al., "SIRT7 regulates hepatocellular carcinoma response to therapy by altering the p53dependent cell death pathway," Journal of Experimental \& Clinical Cancer Research, vol. 38, no. 1, p. 252, 2019.

[56] Z. Tong, M. Wang, Y. Wang et al., "SIRT7 is an RNA-activated protein lysine deacylase," ACS Chemical Biology, vol. 12, no. 1, pp. 300-310, 2017.

[57] H. Shi, Y. Ji, D. Zhang, Y. Liu, and P. Fang, "MicroRNA-3666induced suppression of SIRT7 inhibits the growth of nonsmall cell lung cancer cells," Oncology Reports, vol. 36, no. 5, pp. 3051-3057, 2016.

[58] F. Wang, C. H. Chan, K. Chen, X. Guan, H. K. Lin, and Q. Tong, "Deacetylation of FOXO3 by SIRT1 or SIRT2 leads to Skp2-mediated FOXO3 ubiquitination and degradation," Oncogene, vol. 31, no. 12, pp. 1546-1557, 2012.

[59] S. H. Park, O. Ozden, G. Liu et al., "SIRT2-mediated deacetylation and tetramerization of pyruvate kinase directs glycolysis and tumor growth," Cancer Research, vol. 76, no. 13, pp. 3802-3812, 2016.

[60] L. Xu, Y. Li, L. Zhou et al., "SIRT3 elicited an anti-Warburg effect through HIF1 $\alpha /$ PDK1/PDHA1 to inhibit cholangiocarcinoma tumorigenesis," Cancer Medicine, vol. 8, no. 5, pp. 2380-2391, 2019.

[61] L. Shi, H. Yan, S. An et al., "SIRT5-mediated deacetylation of LDHB promotes autophagy and tumorigenesis in colorectal cancer," Molecular Oncology, vol. 13, no. 2, pp. 358-375, 2019.

[62] J. Wang, Z. Sheng, and Y. Cai, “SIRT6 overexpression inhibits HIF1alpha expression and its impact on tumor angiogenesis in 
lung cancer," International Journal of Clinical and Experimental Pathology, vol. 11, no. 6, pp. 2940-2947, 2018.

[63] J. Cai, Y. Zuo, T. Wang et al., "A crucial role of SUMOylation in modulating Sirt6 deacetylation of $\mathrm{H} 3$ at lysine 56 and its tumor suppressive activity," Oncogene, vol. 35, no. 37, pp. 4949-4956, 2016.

[64] H. Qi, X. Shi, M. Yu et al., "Sirtuin 7-mediated deacetylation of WD repeat domain 77 (WDR77) suppresses cancer cell growth by reducing WDR77/PRMT5 transmethylase complex activity," The Journal of Biological Chemistry, vol. 293, no. 46, pp. 17769-17779, 2018.

[65] J. Jung, L. J. Kim, X. Wang et al., "Nicotinamide metabolism regulates glioblastoma stem cell maintenance. JCI Insight," vol. 2, no. 10, p. 2, 2017.

[66] P. Mierzejewska, T. Gawlik-Jakubczak, P. Jablonska et al., "Nicotinamide metabolism alterations in bladder cancer: preliminary studies," Nucleosides, Nucleotides \& Nucleic Acids, vol. 37, no. 12, pp. 687-695, 2018. 